\title{
Erratum to: Spin-1 diquark contributing to the formation of tetraquarks in light mesons
}

\author{
Hungchong Kim ${ }^{1, a}$, Myung-Ki Cheoun ${ }^{2}$, K. S. Kim ${ }^{3}$ \\ ${ }^{1}$ Research Institute of Basic Science, Korea Aerospace University, Goyang 412-791, Korea \\ 2 Department of Physics, Soongsil University, Seoul 156-743, Korea \\ ${ }^{3}$ School of Liberal Arts and Science, Korea Aerospace University, Goyang 412-791, Korea
}

Received: 27 July 2017 / Accepted: 1 August 2017 / Published online: 14 August 2017

(C) The Author(s) 2017. This article is an open access publication

\section{Erratum to: Eur. Phys. J. C (2017) 77:173 DOI 10.1140/epjc/s10052-017-4736-6}

In the paper above, we have proposed a tetraquark picture with the mixing scheme for the $I_{z}=1$ members of the isovector $(I=1)$ resonances, $a_{0}^{+}(980), a_{0}^{+}(1450)$. In particular, their mass splittings fit relatively well with the hyperfine mass splittings if they are viewed as mixtures of two spin-configurations of diquark-antidiquark constituents, $\left|J, J_{12}, J_{34}\right\rangle=|000\rangle,|011\rangle$, where $J$ is the tetraquark spin, $J_{12}$ the diquark spin, $J_{34}$ the antidiquark spin. The second configuration involving the spin-1 diquark, $|011\rangle$, is found to be an important ingredient in explaining the resonances of our concern in this tetraquark picture. However, the existence of the $|011\rangle$ component requires additional tetraquarks to be found in $J=1$ and $J=2$ resonances with the spin configurations, $\left|J, J_{12}, J_{34}\right\rangle=|111\rangle$ and $|211\rangle$, respectively.

In this erratum, we point out that our assignment of $a_{1}^{+}(1260)$ as a candidate for the $J=1$ tetraquark with the |111) configuration is incorrect because of the $C$-parity for its corresponding member in $I_{z}=0$. Specifically, we would like to demonstrate that the $|111\rangle$ state with $I=1, I_{z}=0$ must have the $C$-parity odd and, in this regard, a relevant candidate for the $|111\rangle$ state should be $b_{1}^{0}(1235)\left(J^{P C}=1^{+-}\right)$ instead of $a_{1}^{0}(1260)\left(J^{P C}=1^{++}\right)$. So its charged member $\left(I=1, I_{z}=1\right)$, which in fact was considered in our paper, must be $b_{1}^{+}$(1235) instead of $a_{1}^{+}(1260)$. Nevertheless, since their experimental masses are almost the same, $M\left[b_{1}(1235)\right]=1229.5 \mathrm{MeV}, M\left[a_{1}(1260)\right]=1230 \mathrm{MeV}$, our discussion in the paper, which is mostly based on the mass splittings, is unaltered except that $a_{1}(1260)$ is replaced with $b_{1}(1235)$. The other tetraquarks with $J=0, J=2$,

The online version of the original article can be found under doi:10.1140/epjc/s10052-017-4736-6. with the spin configurations $|000\rangle,|011\rangle,|211\rangle$, are found to have $C=+$ so their assignments to the physical resonances are not contradictory with their $C$-parity.

To demonstrate that $C|111\rangle=-|111\rangle$ for the isospin member of $I=1, I_{z}=0$, we take the state with $J=$ 1 and the spin projection $M=1$ among three spin states in $|111\rangle$, and we denote this state as $|J M\rangle=|11\rangle$. The same proof can be done for the other spin states, $|J M\rangle=$ $|10\rangle,|1-1\rangle$. The flavor structure of the member $I=1, I_{z}=$ 0 is $\frac{1}{\sqrt{2}}([s u][\bar{s} \bar{u}]-[d s][\bar{d} \bar{s}])$. For our purpose, it would be enough to consider one specific combination of the flavor, $[s u][\bar{s} \bar{u}]$. If we rewrite the state $|J M\rangle=|11\rangle$ with respect to the spins and their projections of diquark and antidiquark, $\left|J_{12} M_{12}\right\rangle_{[s u]}\left|J_{34} M_{34}\right\rangle_{[\bar{s} \bar{u}]}$, we find

$$
\begin{aligned}
|11\rangle= & \frac{1}{\sqrt{2}}\left\{\left|1_{12} 1_{12}\right\rangle_{[s u]}\left|1_{34} 0_{34}\right\rangle_{[\bar{s} \bar{u}]}\right. \\
& \left.-\left|1_{12} 0_{12}\right\rangle_{[s u]}\left|1_{34} 1_{34}\right\rangle_{[\bar{s} \bar{u}]}\right\} .
\end{aligned}
$$

Now it is straightforward to prove that the state above has $C=-$ by applying the charge conjugation [Eq. (2)], exchanging the diquark and antidiquark parts [Eq. (3)], and renaming the dummy indices $12 \leftrightarrow 34$ [Eq. (4)], i.e.,

$$
\begin{aligned}
C|11\rangle= & \frac{1}{\sqrt{2}}\left\{\left|1_{12} 1_{12}\right\rangle_{[\bar{s} \bar{u}]}\left|1_{34} 0_{34}\right\rangle_{[s u]}\right. \\
& \left.-\left|1_{12} 0_{12}\right\rangle_{[\bar{s} \bar{u}]}\left|1_{34} 1_{34}\right\rangle_{[s u]}\right\} \\
= & \frac{1}{\sqrt{2}}\left\{\left|1_{34} 0_{34}\right\rangle_{[s u]}\left|1_{12} 1_{12}\right\rangle_{[\bar{s} \bar{u}]}\right. \\
& \left.-\left|1_{34} 1_{34}\right\rangle_{[s u]}\left|1_{12} 0_{12}\right\rangle_{[\bar{s} \bar{u}]}\right\} \\
= & \frac{1}{\sqrt{2}}\left\{\left|1_{12} 0_{12}\right\rangle_{[s u]}\left|1_{34} 1_{34}\right\rangle_{[\bar{s} \bar{u}]}\right. \\
& \left.-\left|1_{12} 1_{12}\right\rangle_{[s u]}\left|1_{34} 0_{34}\right\rangle_{[\bar{s} \bar{u}]}\right\} \\
= & -|11\rangle .
\end{aligned}
$$

a e-mail: hungchong@kau.ac.kr 
Open Access This article is distributed under the terms of the Creative Commons Attribution 4.0 International License (http://creativecomm ons.org/licenses/by/4.0/), which permits unrestricted use, distribution, and reproduction in any medium, provided you give appropriate credit to the original author(s) and the source, provide a link to the Creative Commons license, and indicate if changes were made.

Funded by SCOAP ${ }^{3}$. 